\section{Government opens consul
mandatory vaccination}

The British Dental Association (BDA) has welcomed the government's move to open a consultation on ending mandatory vaccination but stressed that collateral damage among the workforce remains likely, given the number of colleagues who have already made plans to leave the service and the uncertainty now facing staff in the weeks ahead.

Under the regulations fast-tracked through parliament in December, staff in both NHS and private practice risk dismissal if they are not double jabbed by April, leaving 3 February the effective deadline for a first vaccination. Over 1,000 NHS dentists left the service in the last year, and recruitment and retention problems are already endemic among both dentists and the wider team.

The service is already facing unprecedented backlogs, with over 38 million NHS dental appointments lost since the first lockdown due to the initial suspension of face-to-face care and ongoing infection control measures.

In response to a BDA survey in December, one in ten high street dentists said they were not fully vaccinated, with $9 \%$ reporting they had not had their first jab. Less than two-thirds of respondents believed all dentists operating in their practices were fully vaccinated, with less than half saying their dental nurses, who are integral to the delivery of care, were fully vaccinated. Over $30 \%$ believed dental nurses at their practices would leave as a result of the mandate.

The regulations were subject to a damning report by the House of Lords Secondary Legislation Scrutiny Committee. While an impact assessment was published at the eleventh hour, it simply extrapolated patterns of vaccine hesitancy for primary care dentistry from the wider NHS workforce, and failed to acknowledge the depth of recruitment and retention problems that already exist in the sector. The $\mathrm{BDA}$ is aware that internal consultations in many

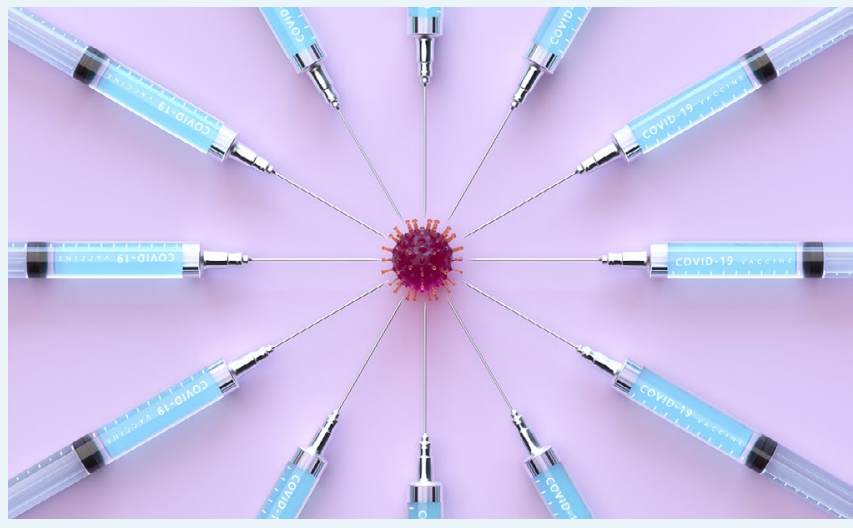

practices began in earnest following the passage of regulations in December, that notices have already been provided by some hesitant staff, and that significant damage has been sustained to working relationships within many close-knit dental teams.

The BDA - like all major healthcare unions - had supported the vaccine programme from the outset, but objected to this policy on the likely impact on workforce sustainability and patient access.

On 31 January, BDA Chair Eddie Crouch said: 'What we needed today was a clean break; instead colleagues now face confusion, and real uncertainty as to where they stand in the days and weeks ahead.

'Mandatory vaccination offers a lesson in how not to make laws. MPs and Peers rightly decried the impossibility of scrutinising fasttracked legislation based on back-of-an-envelope calculations. This service was already haemorrhaging talent, and these rules would have pushed many dental practices over the edge.

'The late return of common sense means millions of patients may still dodge a bullet. However, it remains to be seen how many notices have already been handed in, and what the impact will be on the thousands already reconsidering their futures.'

\title{
New funds allocated to dentistry must be 'just the start'
}

The British Dental Association (BDA) has said new funds allocated to NHS dentistry represent much-needed progress but warned the time-limited package can only be the start when it comes to rebuilding the service and tackling an unprecedented backlog.

On 25 January, NHS England pledged an extra $£ 50$ million for dentists to provide additional urgent care for NHS patients. Funding will be available until the end of the NHS financial year at the end of March and will be paid on a sessional basis.

With practices already struggling to hit gruelling targets, the BDA has stressed the narrow timeline will limit what is achievable in the short term. Since 1 January, contractors must hit $85 \%$ of pre-COVID activity to avoid financial penalties, amid ongoing disruption caused by the Omicron wave. Given major limits on capacity and the difficulties bringing on new staff at short notice, the BDA is not confident the full allocation can be used by the deadline.

Over 38 million NHS appointments have been lost since lockdown as a result of strict infection control protocols, and the resulting backlog will take years to clear. Yet despite calls by the BDA,
Healthwatch England and a growing number of MPs, until now not one penny of the government's multi-billion pound NHS catch-up programme has been allocated to dentistry.

In real terms, government contributions fell by nearly a third between 2010 and 2019. In that period, inflation-busting increases saw the proportion of budget drawn from patient charges surge. Given the cumulative impact of inflation and population growth, it would take an additional allocation of $£ 880$ million of government contributions to the service per annum simply to restore resources to 2010 levels.

Shawn Charlwood, Chair of the BDA's General Dental Practice Committee, said: 'After a decade of cuts, a cash-starved service risks being offered money that can't be spent. Hard-pressed practices are working against the clock, and many will struggle to find capacity ahead of April for this investment to make a difference.

'Until today, not a penny of the government's multi-billion-pound catch-up programme had reached dentistry. This is progress, but must be just the start if we are to rebuild a service millions depend on. 